\title{
Alteration and clinical relevance of PTEN expression and its correlation with survivin expression in epithelial ovarian tumors
}

\author{
LI SUI, YOUYI DONG, YASUO WATANABE, FUMINORI YAMAGUCHI, \\ KATSUYOSHI SUGIMOTO and MASAAKI TOKUDA
}

\author{
Department of Cell Physiology, Faculty of Medicine, Kagawa University, Kagawa, Japan
}

Received September 12, 2005; Accepted November 7, 2005

\begin{abstract}
The tumor suppressor PTEN, phosphatase and tensin homolog on chromosome 10, plays an essential role in regulating signaling pathways involved in cell growth and apoptosis and is inactivated in a wide variety of tumors. Survivin, a member of the inhibitor of apoptosis protein family (IAP), is associated with cell proliferation, and overexpressed in common human tumors. Both PTEN and survivin proteins can regulate cell cycle and apoptosis, but their biological effects are adverse. We have previously investigated the role of survivin expression in epithelial ovarian tumors. In this study, we evaluated the alteration and clinical relevance of PTEN expression and further assessed its correlation with survivin expression in epithelial ovarian tumors. Immunohistochemical analysis was performed in 103 cases of ovarian tumors, and 26 of the 103 cases were evaluated by Western blot analysis. PTEN expression was reduced from benign to malignant ovarian tumors $(\mathrm{p}=0.0003)$, and an inverse correlation between PTEN and survivin was found in benign, borderline, and malignant tumors $(\mathrm{p}=0.004, \mathrm{p}=0.015$ and $\mathrm{p}=0.0005$, respectively). PTEN expression was significantly associated with tumor grade $(\mathrm{p}=0.001)$, histological subtype $(\mathrm{p}=0.037)$, ascites $(\mathrm{p}=0.038)$, and residual disease $(\mathrm{p}=0.0006)$. Kaplan-Meier survival analysis showed that the loss of PTEN expression was significantly associated with poor overall survival $(\mathrm{p}=0.021)$, and patients with PTEN(-)/ survivin(+) expression had the worst prognosis among all phenotypes of PTEN/survivin expression $(\mathrm{p}=0.039)$. Our results suggest that the altered PTEN expression and its inverse correlation with survivin may be involved in the development and progression of ovarian tumors, and the combined detection of PTEN and survivin proteins might be more valuable in the evaluation of malignancy and prognosis in epithelial ovarian tumors.
\end{abstract}

Correspondence to: Dr Masaaki Tokuda, Department of Cell Physiology, Faculty of Medicine, Kagawa University, 1750-1 Ikenobe, Miki-Cho, Kita-Gun, Kagawa 761-0793, Japan

E-mail: tokuda@med.kagawa-u.ac.jp

Key words: PTEN, survivin, ovarian tumor

\section{Introduction}

PTEN, phosphatase and tensin homolog on chromosome 10, was highlighted as a tumor suppressor gene $(1,2)$, which encodes a dual activity phosphatase and is centrally placed to regulate a network of multiple signal transduction pathways involved in cell cycle regulation and cell adhesion properties, including cell proliferation, migration and death (3). PTEN can inhibit cell cycle progression and induce $\mathrm{G}_{1}$ arrest through negatively regulating the PI3K (phosphatidylinositol 3-kinase)/ AKT (protein kinase B) signaling pathway (4-7). A loss of PTEN function leads to increased PI3K/AKT activity and subsequent increased cell proliferation, reduced apoptosis, altered migration, and increased size in all phenotypes that favor tumorigenesis (8). PTEN mutations or deletions have been identified in a large number of primary malignant tumors including glioblastomas, and breast, gastric, esophageal and endometrial cancer, and also showed that reduced PTEN protein was associated with poor prognosis (9-15). The PI3K/AKT pathway is a major driving force in human cancer, and a common way of stimulating the PI3K/AKT pathway occurs through inactivation of the PTEN tumor suppressor. PI3K has been implicated not only in cell survival signaling, but also in the inhibition of apoptosis by inactivation of cell death proteins such as BAD14, and a death effector protein, caspase-9 (16).

Survivin, a member of the IAP family, is found to be expressed in fetal tissues and many cancers but undetectable in most normal adult tissues (17). Survivin can suppress apoptosis induced by Fas, Bax, caspases, and anticancer drugs (18). Our study (19) and other studies (20-25) have indicated that survivin is associated with the aggressiveness of tumors and unfavorable clinical outcomes. Additionally, Kim et al have reported that inhibition of the PI3K/AKT pathway down-regulates survivin expression in neuroblastomas (26), and activation of the PI3K/AKT pathway can increase survivin expression (27-30). These findings raised the question whether increased survivin expression in ovarian carcinoma is related to reduced PTEN expression. To gain better insight, we investigated the correlation of PTEN and survivin expression, as well as the clinical outcomes of PTEN in a group of epithelial ovarian tumors.

\section{Materials and methods}

Tumor specimens. Formalin-fixed, paraffin-embedded blocks of ovarian tumor tissues from 77 patients ( 32 malignant, 23 

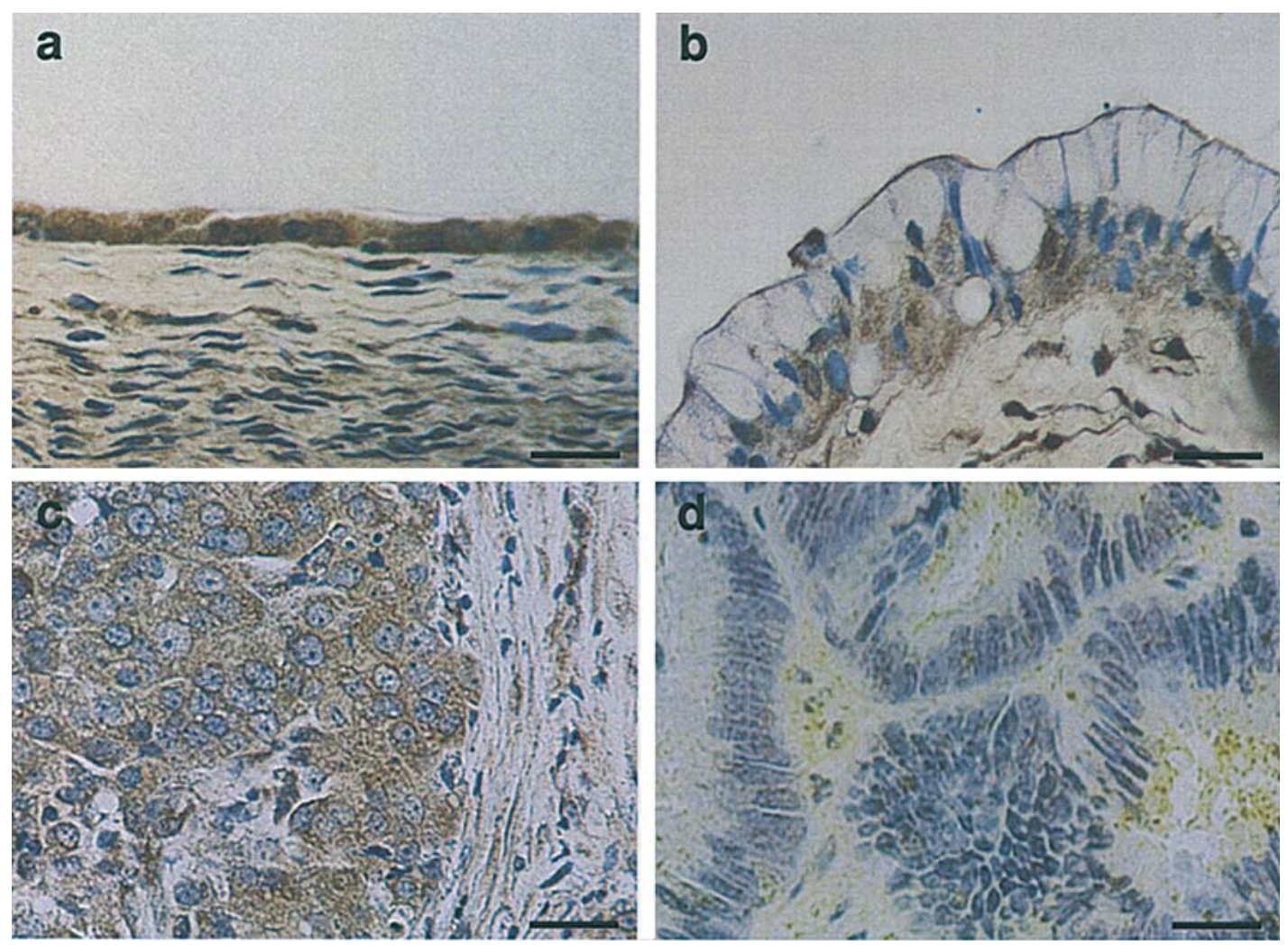

Figure 1. Immunohistochemical staining patterns of PTEN in ovarian tumors. (a) Positive staining was shown in benign ovarian tumor cells. (b) Positive staining was shown in borderline ovarian tumor cells. (c) Positive staining was shown in ovarian carcinoma cells. (d) Negative staining was shown in ovarian carcinoma cells. Scale bars, $30 \mu \mathrm{m}$.

borderline, and 22 benign ovarian tumors) were obtained at the Department of Perinatology and Gynecology, Faculty of Medicine, Kagawa University from 1985 to 1996. Fresh ovarian tumor samples $(n=26 ; 15$ malignant and 11 benign ovarian tumors) were obtained at the Department of Perinatology and Gynecology, Faculty of Medicine, Kagawa University and Department of Obstetrics and Gynecology of Takamatsu Red Cross Hospital from 1997 to 1998. After surgical resection, each fresh tumor specimen was immediately washed and cut out around necrotic tissue, then divided into two portions: one was instantly frozen for protein extraction and the other was formalin-fixed and paraffin-embedded for routine and immunohistochemical investigation. Specimens consisted of 33 benign, 23 borderline and 47 malignant ovarian tumors. The median age of the 47 ovarian carcinoma patients was 49 years (range, 16-77), and 16 patients were in stage I, 3 in stage II, 16 in stage III, and 12 in stage IV according to the International Federation of Gynecology and Obstetrics (FIGO) classification. The histological classification of tumors was carried out according to the WHO system with 21 well-differentiated $\left(\mathrm{G}_{1}\right), 13$ moderately differentiated $\left(\mathrm{G}_{2}\right)$ and 13 poorly differentiated (G3, including 2 undifferentiated) cases. Among the 47 patients with ovarian carcinomas, none received preoperative chemotherapy or radiotherapy. All patients received postoperative, platinum-based chemotherapy, but no radiotherapy. Follow-up data were available for all patients.

Immunohistochemistry. Paraffin sections ( $4 \mu \mathrm{m}$ thickness) were deparaffinized and rehydrated. Endogenous peroxidase activity was blocked using $0.3 \%$ hydrogen peroxide $(30 \mathrm{~min})$.
To reduce nonspecific binding, the sections were incubated with $10 \%$ horse serum for $60 \mathrm{~min}$ at room temperature. The antigen retrieval procedure was performed by microwave oven heating, 3 times for $3 \mathrm{~min}$ each in $10 \mathrm{mM}$ citric acid at pH 6.0. The sections were incubated overnight at $4{ }^{\circ} \mathrm{C}$ with mouse anti-PTEN monoclonal antibody (Santa Cruz Biotechnology, Santa Cruz, CA, USA; 1:100), and a corresponding section for each case was incubated with nonimmunized mouse serum as a negative control. Immunostaining was performed by the ABC (avidin-biotin peroxidase complex) method using a Vectastain ABC kit (Vector Laboratories, Burlingame, CA, USA). Peroxidase activity was detected using DAB (3,3diaminobenzidine) as the chromogen and Mayer's hematoxylin as the counterstain. All of the samples (formalin-fixed and fresh) were handled using the same method and antibody.

The immunoreactive cells were independently evaluated by two of our members (L.S. and Y.D.), who were unaware of the clinicopathological factors and clinical outcome of patients. At least 10 high-power fields were chosen randomly, and 1000 cells were counted. PTEN immunoreactivity was estimated according to the cytoplasmic staining intensity and percentage of positivity. The staining intensity was divided into 3 categories: 0 , absent; 1 , weak; and 2, moderate to strong. The quantity of stained cells was scored as: $1,<10 \%$; $2,10-50 \%$; and $3,>50 \%$. A total score of $\leq 4$ was defined as reduced PTEN expression and denoted as (-).

Western blot analysis. Approximately $0.5 \mathrm{~g}$ of tissue from each fresh tumor sample was homogenized and lysed in $2.5 \mathrm{ml}$ of lysis buffer [1\% NP-40, $150 \mathrm{mM} \mathrm{NaCl}, 50 \mathrm{mM}$ $\mathrm{NaF}, 20 \mathrm{mM}$ Tris- $\mathrm{HCl}$ (pH 7.5), 5 mM EDTA, 1 mM Na $\mathrm{VO}_{4}$, 
Table I. PTEN expression in benign, borderline, and malignant ovarian tumors.

\begin{tabular}{lccrr}
\hline & & \multicolumn{2}{c}{ PTEN expression } & \\
\cline { 3 - 4 } & Total & $+(\%)$ & $-(\%)$ & p-value \\
\hline Benign & 33 & $29(87.9)$ & $4(12.1)$ & $<0.0001^{\text {a }}$ \\
Borderline & 23 & $16(69.6)$ & $7(30.4)$ & $0.0500^{\mathrm{b}}$ \\
Malignant & 47 & $21(44.7)$ & $26(55.3)$ & 0.0003 \\
\hline
\end{tabular}

${ }^{\mathrm{a} B e n i g n}$ vs. malignant. ${ }^{\mathrm{b} B}$ orderline vs. malignant.

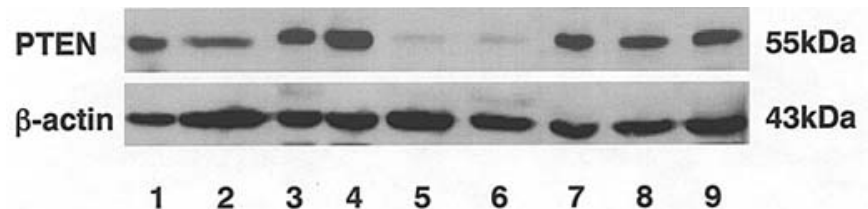

Figure 2. Western blot analysis of PTEN in benign and malignant ovarian tumors. PTEN expression was observed in all benign tumors (lanes 1-4). General or reduced PTEN expression was observed in malignant tumors (lanes 5-8). NGF-treated PC12 cells were used as a positive control (lane 9) (43). The amount of $B$-actin was demonstrated at a constant level among the samples.

$10 \mu \mathrm{M} \mathrm{Na}_{2} \mathrm{MnO}_{4}, 1 \mathrm{mM}$ PMSF, $10 \mu \mathrm{g} / \mathrm{ml}$ leupeptin, and $1 \%$ aprotinin]. The lysates were centrifuged at $100,000 \times \mathrm{g}$ for $1 \mathrm{~h}$ at $4^{\circ} \mathrm{C}$ and the supernatant was stored at $-80^{\circ} \mathrm{C}$ until further analysis. Extracts equivalent to $200 \mu \mathrm{g}$ of the total protein were separated by $12 \%$ SDS-polyacrylamide gel, then transferred to polyvinylidene fluoride membranes (Immobilon-P, Millipore, Bedford, MA, USA). The membranes were blocked in TBS containing 5\% nonfat dried milk, $10 \%$ sheep serum and $0.1 \%$ Tween-20, then probed by monoclonal antibody against PTEN (1:200), and against $\beta$-actin (1:500; Sigma, St. Louis, MO, USA) in PBS containing 5\% bovine serum. After several washes with TBS, membranes were probed with a horseradish peroxidase-conjugated anti-mouse IgG (Dako, Kyoto, Japan), and proteins were detected using an enhanced chemiluminescence (ECL) system (Amersham, Tokyo, Japan).

Statistical analysis. The correlation between PTEN and clinicopathological parameters was assessed using Pearson's $\chi^{2}$ test. The Spearman rank correlation was used to determine whether there was a correlation between PTEN and survivin expression. Overall survival was calculated using the Kaplan-Meier method, and comparison between groups was performed with the log-rank test. The Cox proportional hazards regression model was used to estimate the relative risk ratio (RR) of death in the $95 \%$ confidence interval (CI) and identify the variables associated with overall survival. Statistical significance was set at $p<0.05$. Statistical analyses were run using JMP software version 3.2.5 (SAS Institute Inc., Cary, NC).

\section{Results}

Expression of PTEN in ovarian tumors. Immunohistochemical analysis revealed that PTEN expression was located mainly
Table II Correlation between PTEN expression and clinicopathological parameters.

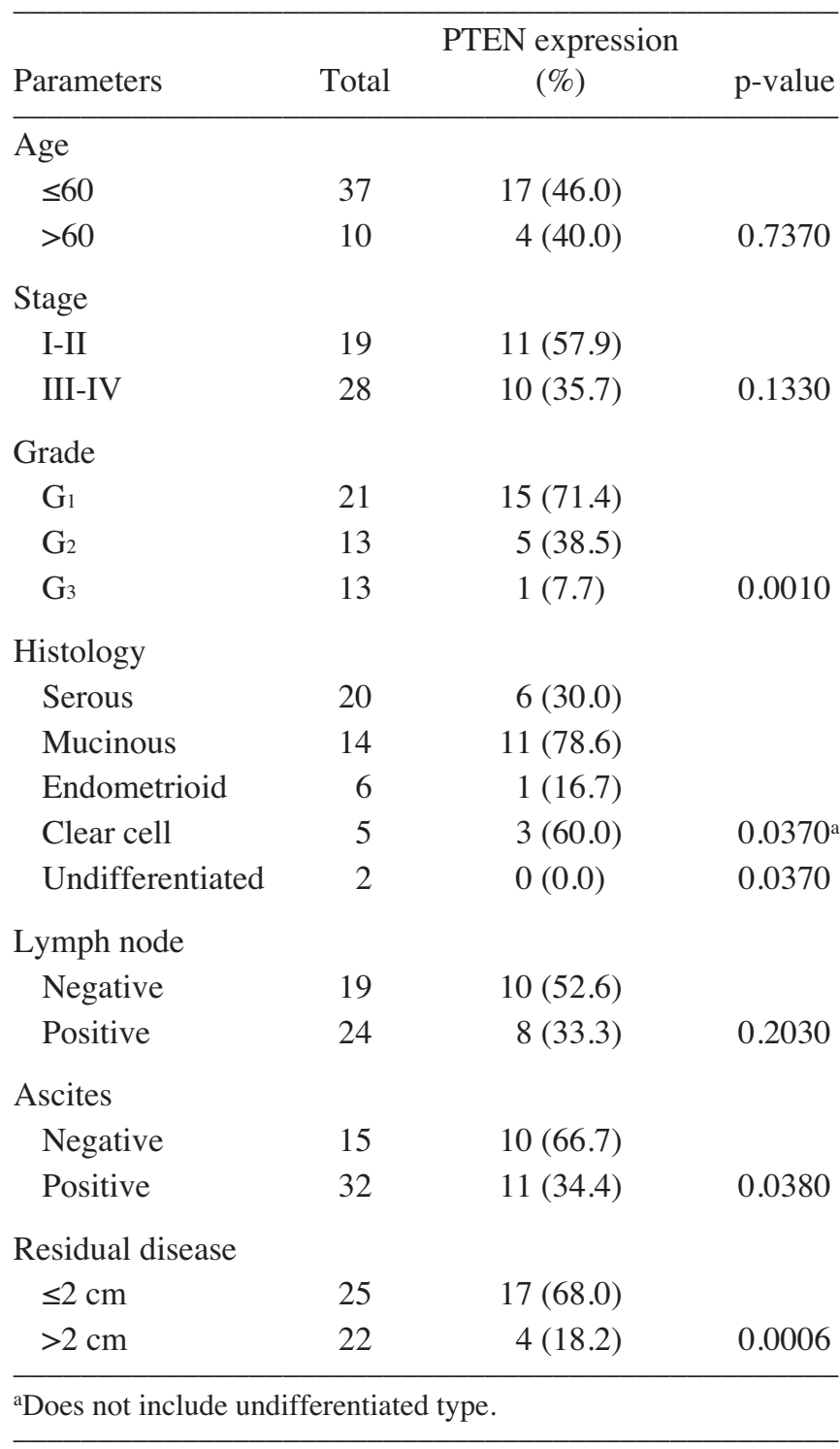

in the cytoplasm of ovarian tumor cells (Fig. 1a-c), and weak nuclear staining was also shown; an example of negative expression is shown in Fig. 1d. Reduced PTEN expression was detected in $12.1 \%$ (4 of 33) of benign tumors, 30.4\% (7 of 23 ) of borderline tumors, and $55.3 \%$ (26 of 47) of malignant tumors (Table I). The PTEN expression in malignant tumors was significantly lower than that in benign tumors $(\mathrm{p}<0.0001)$, and borderline lower than that in borderline tumors $(\mathrm{p}=0.05)$. The total tendency of PTEN expression was decreased from benign to borderline to malignant tumors $(\mathrm{p}=0.0003)$.

To confirm the specificity of the immunohistochemical results, Western blot analysis was carried out in 11 benign and 15 malignant ovarian tumors, in which freshly frozen materials were available. An example of Western blot analysis is shown in Fig. 2. An immunoreactive band of PTEN was observed in both benign and malignant ovarian tumors (lanes 1-4, 7 and 8), and 2 cases of malignant tumor showed reduced PTEN expression (lanes 5 and 6).

Correlation of PTEN expression with survivin. Our previous data showed survivin expression in $21.2 \%$ of benign, $47.8 \%$ 

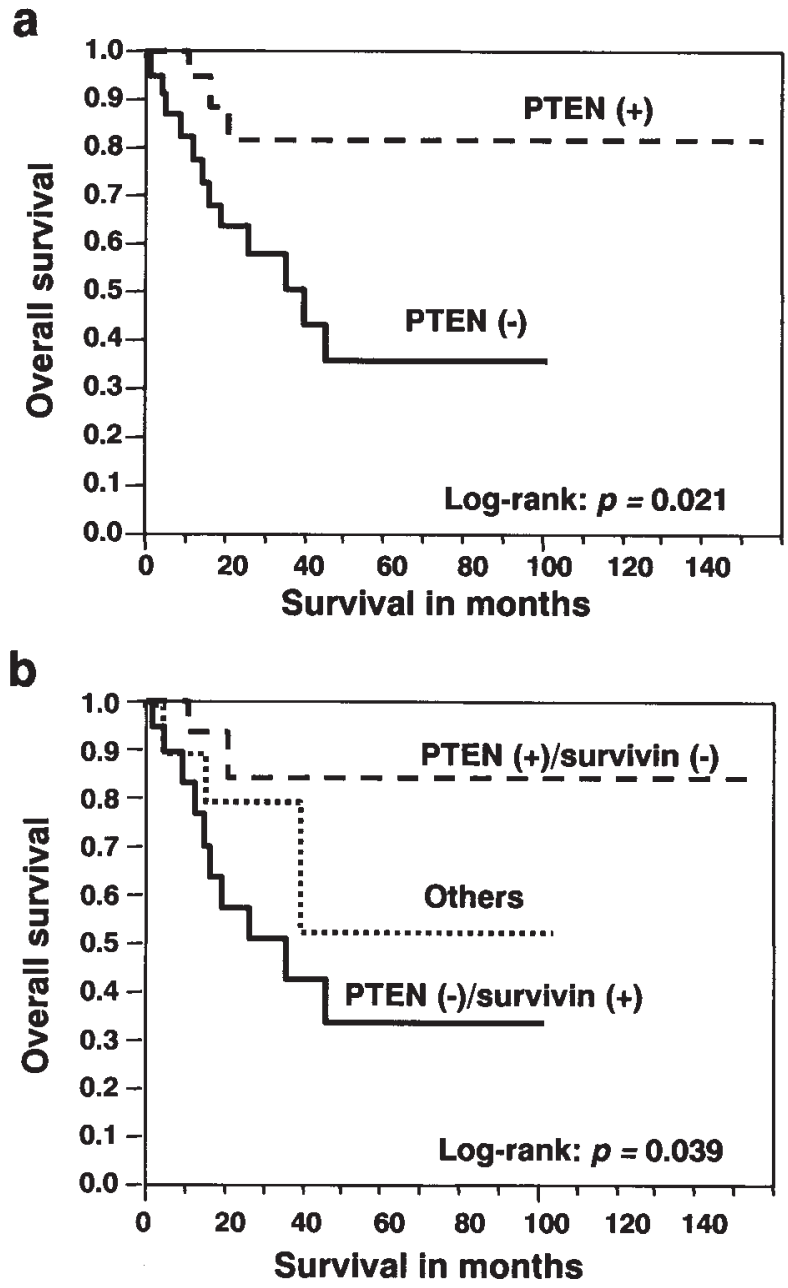

Figure 3. Kaplan-Meier survival curves of patients with ovarian carcinoma. (a) Overall survival according to PTEN expression ( $\mathrm{p}=0.021)$. (b) Overall survival according to PTEN/survivin phenotypes $(\mathrm{p}=0.039)$.

in borderline, and $51.1 \%$ in malignant tumors, respectively (19). The correlation between PTEN and survivin expression was investigated using Spearman's rank correlation. A negative correlation between both proteins was identified in benign (correlation coefficient, $-0.49 ; \mathrm{p}=0.004$ ), borderline (correlation coefficient, $-0.5 ; \mathrm{p}=0.015$ ), and malignant tumors (correlation coefficient, $-0.49 ; \mathrm{p}=0.0005$ ). Patients with PTEN(-)/survivin(+) expression showed 9.1\%, 26.1\% and $42.6 \%$ benign, borderline, and malignant tumors, respectively, and the ratio was increased with malignancy $(\mathrm{p}=0.005)$. In contrast, the patients with PTEN(+)/survivin(-) expression showed $75.8 \%, 47.8 \%$, and $36.2 \%$ benign, borderline, and malignant tumors, respectively, and the ratio showed a decreasing tendency $(\mathrm{p}=0.002)$.

Correlation between PTEN expression and clinicopathological parameters. We evaluated the correlation between PTEN expression and clinicopathological parameters such as tumor grade, clinical stage, histology, lymph node status, etc. A significant correlation between PTEN expression and tumor grade $(\mathrm{p}=0.001)$, histology $(\mathrm{p}=0.037)$, ascites $(\mathrm{p}=0.038)$, and residual disease $(\mathrm{p}=0.0006)$ was revealed (Table II).
Survival analysis. The median follow-up time for all of the ovarian carcinoma patients was 24 months (range, 2-156). At the end-point of the follow-up, 32 patients survived with a median follow-up time of 27.5 months (range, 4-156), and 15 had died of ovarian cancer after a median follow-up of 17 months (range, 2-46). Kaplan-Meier analysis showed that reduced PTEN expression has a significant adverse effect on overall survival ( $\mathrm{p}=0.021$; Fig. $3 \mathrm{a}$ ). Our previous study showed that increased survivin expression was significantly associated with poor overall survival ( $\mathrm{p}=0.037)(19)$. In the current study, we further analyzed the combined phenotypes of PTEN and survivin proteins. The patients with PTEN(-)/survivin(+) expression had the worst overall survival among all phenotypes of PTEN/survivin ( $p=0.039$; Fig. 3b).

Multivariate Cox regression analysis of PTEN expression was performed together with other factors showing prognostic significance of univariate analysis such as clinical stage (RR, 1.98; CI, 1.11-4.16; $\mathrm{p}=0.019)$, tumor grade (RR, 1.94; CI, $1.13-3.70 ; \mathrm{p}=0.015)$, lymph node status (RR, 2.41; CI, 1.25$6.15 ; \mathrm{p}=0.007)$, and residual disease (RR, 2.79; CI, 1.575.87; $\mathrm{p}=0.0003$ ). Although PTEN expression was not defined as an independent prognostic factor, the patients with reduced PTEN expression have the highest relative risk ratio of death (RR, 3.96; CI, 1.26-7.41; $\mathrm{p}=0.017$ ).

\section{Discussion}

In the present study, PTEN expression was found in $87.9 \%$, $69.6 \%$ and $44.7 \%$ of benign, borderline, and malignant ovarian tumors, respectively. A significant difference was observed between benign and malignant ovarian tumors $(p<0.0001)$. PTEN expression was gradually diminished from benign to borderline to malignant tumors $(\mathrm{p}=0.0003)$. The finding suggested that down-regulated PTEN expression might be closely associated with malignant transformation of ovarian tumors. Although no immunohistochemical data were available for PTEN expression simultaneously in benign, borderline, and malignant ovarian tumors, a correlation between carcinogenesis and a lack of PTEN expression has been demonstrated in ovarian carcinoma $(31,32)$ and other cancers. Diminished or absent PTEN expression was reported in $66 \%$ of endometrial carcinoma (33), $38 \%$ of breast cancer (34), $27.2 \%$ of prostate cancer (35), and $24 \%$ of non-small cell lung cancer (36). Consequently, the alteration of PTEN expression might be a major general event in the progression of carcinomas of different origins.

Survivin, an inhibitor of apoptosis protein, was investigated in our previous study, and found to play a crucial role in the progression of ovarian carcinomas (19). It is recognized that $\mathrm{PI} 3 \mathrm{~K} / \mathrm{AKT}$ pathway-dependent cell survival is negatively regulated by PTEN $(8,37)$. PTEN and survivin are two inverse factors of apoptosis; thus, we attempted to investigate whether PTEN and survivin have a relationship in ovarian tumors. It was firstly found that PTEN expression was negatively correlated with survivin expression in the progression of ovarian tumors, and cases with PTEN(-)/ survivin(+) expression gradually increased from benign to malignant tumors $(\mathrm{p}=0.005)$; in contrast, cases with PTEN(+)/survivin(-) expression gradually decreased from benign to malignant tumors $(\mathrm{p}=0.002)$. Previous studies 
(26-30) revealed that the PI3K/AKT pathway could modulate apoptosis by a mechanism involving the PI3K/AKT pathway activation, subsequent up-regulation of survivin, and the suppression of caspase- 9 activity called the AKT/survivin pathway $(28,30)$. PTEN is known to regulate the PI3K/AKT pathway by removing the third phosphate from the inositol ring of the PIP3 second messenger (38-41), consequently inactivating the PI3K/AKT signaling pathway. From this, we can presume that PTEN might modulate the survivin level by the PI3K/AKT pathway. Ovarian tumors lacking PTEN expression have high survivin expression, thus intensifying the cell survival signal transduction pathway and promoting the progression of ovarian tumors.

In addition, we evaluated the correlation between PTEN expression and clinicopathological parameters. Reduced PTEN expression was found to be significantly associated with increasing tumor grade, ascites, and residual disease. The results are supported by the fact that mammalian cells lacking PTEN can promote proliferation, reduce apoptosis, alter migration, and increase size, which might be responsible for conferring the local invasion of tumors (8). We also found that PTEN expression was significantly lower in the endometrioid histological subtype than other subtypes, which is consistent with the report of Obata et al, who documented that lack of PTEN expression was frequently observed in endometrioid but not serous or mucinous ovarian carcinomas (42). Our finding therefore supports that the molecular pathogenic mechanism of endometrioid ovarian carcinoma is different than other histological subtypes, and PTEN might play an important role in the etiology of the endometrioid subtype.

The possible role of PTEN protein on the prognosis of ovarian tumor patients remains unclear. Currently, prognostic evaluation is primarily based on the traditional method that includes clinical stage, tumor grade, lymph node status, etc. However, in past decades, tumor markers have been demonstrated to be clinically helpful in detecting various human tumors. Studies on PTEN expression in breast, brain, gastric, esophageal, and endometrial cancer indicated that reduced PTEN protein was associated with poor prognosis (9-15). In this study, survival analysis by the Kaplan-Meier method revealed that patients lacking PTEN expression have a significantly shorter overall survival and show an approximate 4-fold higher relative risk of death (RR, 3.96; $\mathrm{p}=0.017)$. This evidence suggests that a low PTEN level confers a more aggressive phenotype of ovarian tumor cells and indicates that evaluation of the PTEN level may provide a significant prognostic implication in epithelial ovarian carcinomas. Schondorf et al have demonstrated that declining PTEN expression results in a shortened progression-free interval by assessing the PTEN content in both the primary and recurrent ovarian cancer specimens from each patient (32). We also performed a survival analysis of survivin in epithelial ovarian tumors, and found that patients with survivin overexpression had a significantly decreased overall survival (19). To verify whether the combined analysis of PTEN and survivin can provide a more valuable implication in the prognostic evaluation of ovarian carcinoma, a combined phenotype of these two proteins was further examined. We found that patients with PTEN(-)/survivin(+) expression had the worst overall survival among all phenotypes of PTEN/ survivin expression. Since the clinical outcome of ovarian carcinoma patients might be difficult to predict, the combined evaluation of PTEN/survivin can further increase the availability of prognostic information, and the identification of tumors with specific immunophenotypes may prove valuable in the future when selecting patients for experimental treatment protocols.

In conclusion, PTEN expression is negatively associated with survivin expression in the progression of ovarian tumors, and the alteration of PTEN expression is likely an important molecular event in ovarian tumorigenesis. Although PTEN expression cannot be recognized as an independent prognostic factor, the immunohistochemical evaluation of PTEN expression may provide additional prognostic information. Furthermore, the combined detection of PTEN/survivin proteins might be the most reliable indication of prognosis for epithelial ovarian carcinoma patients.

\section{Acknowledgements}

We thank Drs. Masayuki Ohno (Department of Perinatology and Gynecology, Faculty of Medicine, Kagawa University, Kagawa, Japan), Teruo Inohara and Masaki Goto (Department of Obstetrics and Gynecology of Takamatsu Red Cross Hospital, Kagawa, Japan) for providing the tumor specimens. This work was supported by a Grant-in-Aid for Scientific Research from the Ministry of Education, Culture, Sports, Science and Technology of Japan.

\section{References}

1. Li J, Yen C, Liaw D, et al: PTEN, a putative protein tyrosine phosphatase gene mutated in human brain, breast, and prostate cancer. Science 275: 1943-1947, 1997.

2. Steck PA, Pershouse MA, Jasser SA, et al: Identification of a candidate tumor suppressor gene, MMAC1 at chromosome 10q23.3 that is mutated in multiple advanced cancers. Nat Genet 15: 356-362, 1997.

3. Tamura M, Gu J, Takino T and Yamada KM: Tumor suppressor PTEN inhibition of cell invasion, migration, and growth: differential involvement of focal adhesion kinase and p130cas. Cancer Res 59: 4342-4349, 1999.

4. Sakudra A, Hamada H, Fukushige S, et al: Adenovirus mediated delivery of the PTEN gene inhibits cell growth by induction of apoptosis in endometrial cancer. Int J Oncol 15: 1069-1074, 1999.

5. Cheney IW, Neuteboom ST, Vaillancourt MT, Ramachandra M and Bookstein R: Adenovirus-mediated gene transfer of MMAC1/PTEN to glioblastoma cells inhibits $S$ phase entry by the recruitment of $\mathrm{p} 27^{\mathrm{KIP} 1}$ into cyclin E/CDK2 complexes. Cancer Res 59: 2318-2323, 1999.

6. Li DM and Sun H: PTEN/MMAC1/TEP1 suppresses the tumorigenicity and induces G1 cell cycle arrest in human glioblastoma cells. Proc Natl Acad Sci USA 95: 15406-15411, 1998.

7. Weng LP, Smith WM, Dahia PL, Ziebold U, Gil E, Lees JA and Eng C: PTEN suppresses breast cancer cell growth by phosphatase activity-dependent G1 arrest followed by cell death. Cancer Res 59: 5808-5814, 1999.

8. Stambolic V, Suzuki A, de la Pompa JL, et al: Negative regulation of PKB/Akt-dependent cell survival by the tumor suppressor PTEN. Cell 95: 29-39, 1998.

9. Depowski PL, Rosenthal SI and Ross JS: Loss of expression of the PTEN gene protein product is associated with poor outcome in breast cancer. Mod Pathol 14: 672-676, 2001.

10. Sano T, Lin H, Chen X, et al: Differential expression of MMAC/PTEN in glioblastoma multiforme: relationship to localization and prognosis. Cancer Res 59: 1820-1824, 1999.

11. Lee HS, Lee HK, Kim HS, Yang HK and Kim WH: Tumour suppressor gene expression correlates with gastric cancer prognosis. J Pathol 200: 39-46, 2003. 
12. Tachibana M, Shibakita M, Ohno S, et al: Expression and prognostic significance of PTEN product protein in patients with esophageal squamous cell carcinoma. Cancer 94: 1955-1960, 2002.

13. Terakawa N, Kanamori Y and Yoshida S: Loss of PTEN expression followed by Akt phosphorylation is a poor prognostic factor for patients with endometrial cancer. Endocr Relat Cancer 10: 203-208, 2003.

14. Risinger JI, Hayes AK, Berchuck A and Barett JC: PTEN/ MMAC1 mutations in endometrial cancers. Cancer Res 57: 4736-4738, 1997.

15. Tashiro H, Blazes MS, Wu R, et al: Mutations in PTEN are frequent in endometrial carcinoma but rare in other common gynecological malignancies. Cancer Res 57: 3935-3940, 1997.

16. Cardone MH, Roy N, Stennicke HR, et al: Regulation of cell death protease caspase- 9 by phosphorylation. Science 282: 1318-1321, 1998 .

17. Ambrosini G, Adida C and Altieri DC: A novel anti-apoptosis gene, survivin, expressed in cancer and lymphoma. Nat Med 3: 917-921, 1997.

18. Tamm I, Wang Y, Sausville E, Scudiero DA, Vigna N, Oltersdorf T and Reed JC: IAP family protein survivin inhibits caspase activity and apoptosis induced by Fas (CD95), Bax, caspases, and anticancer drugs. Cancer Res 58: 5315-5320, 1998.

19. Sui L, Dong Y, Ohno M, Watanabe Y, Sugimoto K and Tokuda M: Survivin expression and its correlation with cell proliferation and prognosis in epithelial ovarian tumors. Int $\mathbf{J}$ Oncol 21: 315-320, 2002.

20. Adida C, Berrebi D, Peuchmaur M, Reyes-Mugica M and Altieri DC: Anti-apoptosis gene, survivin, and prognosis of neuroblastoma. Lancet 351: 882-883, 1998.

21. Kawasaki H, Altieri DC, Lu CD, Toyoda M, Tenjo T and Tanigawa N: Inhibition of apoptosis by survivin predicts shorter survival rates in colorectal cancer. Cancer Res 58: 5071-5074, 1998.

22. Sarela AI, Macadam RC, Farmery SM, Markham AF and Guillou PJ: Expression of the antiapoptosis gene, survivin, predicts death from recurrent colorectal carcinoma. Gut 46: 645-650, 2000.

23. Monzo M, Rosell R and Felip E, et al: A novel anti-apoptosis gene: Re-expression of survivin messenger RNA as a prognosis marker in non-small-cell lung cancers. J Clin Oncol 17: 2100-2104, 1999.

24. Tanaka K, Iwamoto S, Gon G, Nohara T, Iwamoto M and Tanigawa N: Expression of survivin and its relationship to loss of apoptosis in breast carcinomas. Clin Cancer Res 6: 127-134, 2000.

25. Kato J, Kuwabara Y and Mitani M, et al: Expression of survivin in esophageal cancer: correlation with the prognosis and response to chemotherapy. Int J Cancer (Pred Oncol) 95: 92-95, 2001.

26. Kim S, Kang J, Qiao J, Thomas RP, Evers BM and Chung DH: Phosphatidylinositol 3-kinase inhibition down-regulates survivin and facilitates TRAIL-mediated apoptosis in neuroblastomas. J Pediatr Surg 39: 516-521, 2004

27. Carter BZ, Milella M, Altieri DC and Andreeff M: Cytokineregulated expression of survivin in myeloid leukemia. Blood 197: 2784-2790, 2001.
28. Papapetropoulos A, Fulton D, Mahboubi K, et al: Angiopoietin-1 inhibits endothelial cell apoptosis via the Akt/survivin pathway. J Biol Chem 275: 9102-9105, 2000.

29. Ohashi H, Takagi H, Oh H, et al: Phosphatidylinositol 3-kinase/ Akt regulates angiotensin II-induced inhibition of apoptosis in microvascular endothelial cells by governing survivin expression and suppression of caspase-3 activity. Circ Res 94: 785-793, 2004.

30. Fornaro M, Plescia J, Chheang S, et al: Fibronectin protects prostate cancer cells from tumor necrosis factor-alpha-induced apoptosis via the AKT/survivin pathway. J Biol Chem 278: 50402-50411, 2003

31. Kurose K, Zhou XP, Araki T, Cannistra SA, Maher ER and Eng C: Frequent loss of PTEN expression is linked to elevated phosphorylated Akt levels, but not associated with p27 and cyclin D1 expression, in primary epithelial ovarian carcinomas. Am J Pathol 158: 2097-2106, 2001.

32. Schondorf T, Gohring UJ, Roth G, et al: Time to progression is dependent on the expression of the tumour suppressor PTEN in ovarian cancer patients. Eur J Clin Invest 33: 256-260, 2003.

33. An HJ, Lee YH, Cho NH, Shim JY, Kim JY, Lee C and Kim SJ: Alteration of PTEN expression in endometrial carcinoma is associated with down-regulation of cyclin-dependent kinase inhibitor, p27. Histopathology 41: 437-445, 2002.

34. Bose S, Crane A, Hibshoosh H, Mansukhani M, Sandweis L and Parsons R: Reduced expression of PTEN correlates with breast cancer progression. Hum Pathol 33: 405-409, 2002.

35. Halvorsen OJ, Haukaas SA and Akslen LA: Combined loss of PTEN and p27 expression is associated with tumor cell proliferation by Ki-67 and increased risk of recurrent disease in localized prostate cancer. Clin Cancer Res 9: 1474-1479, 2003.

36. Soria JC, Lee HY, Lee JI, et al: Lack of PTEN expression in nonsmall cell lung cancer could be related to promoter methylation. Clin Cancer Res 8: 1178-1184, 2002.

37. Leslie NR and Downes CP: PTEN: the down side of PI 3-kinase signalling. Cell Signal 14: 285-295, 2002.

38. Sulis ML and Parsons R: PTEN: from pathology to biology. Trends Cell Biol 13: 478-483, 2003.

39. Luo J, Manning BD and Cantley LC: Targeting the PI3K-Akt pathway in human cancer: rationale and promise. Cancer Cell 4: 257-262, 2003.

40. Sansal I and Sellers WR: The biology and clinical relevance of the PTEN tumor suppressor pathway. J Clin Oncol 22: 2954-2963, 2004.

41. Parsons R: Human cancer, PTEN and the PI-3 kinase pathway. Semin Cell Dev Biol 15: 171-176, 2004.

42. Obata K, Morland SJ, Watson RH, Hitchcock A, Thomas EJ, Chenevix-Trench $\mathrm{G}$ and Campbell IG: Frequent PTEN/MMAC mutations in endometrioid, but not serous or mucinous epithelial ovarian tumors. Cancer Res 58: 2095-2097, 1998.

43. Lachyankar MB, Sultana N, Schonhoff CM, et al: A role for nuclear PTEN in neuronal differentiation. J Neurosci 20: 1404-1413, 2000. 